\title{
Subregion spectrum form factor via pseudoentropy
}

\author{
Kanato Goto, ${ }^{1, *}$ Masahiro Nozaki ${ }^{1,2, \uparrow}$ and Kotaro Tamaoka $\oplus^{3, \$}$ \\ ${ }^{1}$ RIKEN Interdisciplinary Theoretical and Mathematical Sciences (iTHEMS), \\ Wako, Saitama 351-0198, Japan \\ ${ }^{2}$ Kavli Institute for Theoretical Sciences and CAS Center for Excellence in Topological Quantum \\ Computation, University of Chinese Academy of Sciences, Beijing 100190, China \\ ${ }^{3}$ Department of Physics, College of Humanities and Sciences, Nihon University,
} Sakura-josui, Tokyo 156-8550, Japan

(Received 8 September 2021; accepted 15 November 2021; published 7 December 2021)

\begin{abstract}
We introduce a subsystem generalization of the spectral form factor via pseudoentropy, the vonNeumann entropy for the reduced transition matrix. We consider a transition matrix between the thermofield double state and its time-evolved state in two-dimensional conformal field theories, and study the time dependence of the pseudoentropy for a single interval. We show that the real part of the pseudoentropy behaves similarly to the spectral form factor; it starts from the thermal entropy, initially drops to the minimum, then it starts increasing, and then finally it approaches the vacuum entanglement entropy. We also study the theory dependence of its behavior by considering theories on a compact space.
\end{abstract}

DOI: 10.1103/PhysRevD.104.L121902

\section{INTRODUCTION}

Quantum entanglement is being studied extensively in several fields such as high-energy physics, condensed matter physics, and quantum information theory.

In high-energy physics, recent studies in AdS/CFT correspondence [1] (AdS/CFT) revealed that information of the geometry in anti-de Sitter (AdS) is encoded in the entanglement structure of the dual conformal field theory (CFT) state, which can be studied using quantuminformation-theoretic quantities, such as the entanglement entropy $[2,3]$.

In condensed matter physics, quantum-informationtheoretic quantities that measure the bipartite and tripartite entanglement have been used to study nonequilibrium phenomena, such as quantum thermalization and information scrambling [4-8]. Moreover, it was found that the spectrum of the reduced density matrix, also called entanglement spectrum, is useful to diagnose quantum phases of matter $[9,10]$. However, the entanglement spectra in quantum field theories are rarely computable analytically [11].

In this paper, we introduce a quantity that characterizes the entanglement spectra of general subregions. In Refs. [12,13],

\footnotetext{
kanato.goto@ riken.jp

masahiro.nozaki@riken.jp

tamaoka.kotaro@nihon-u.ac.jp
}

Published by the American Physical Society under the terms of the Creative Commons Attribution 4.0 International license. Further distribution of this work must maintain attribution to the author(s) and the published article's title, journal citation, and DOI. Funded by SCOAP. the following quantities that characterize the energy spectrum have been proposed

$$
\begin{gathered}
\left|\frac{Z(\beta+i t)}{Z(\beta)}\right|^{2}=\frac{1}{Z(\beta)^{2}} \sum_{n, m} e^{-\beta\left(E_{m}+E_{n}\right)} e^{i t\left(E_{m}-E_{n}\right)}, \\
Z(\beta+i t) \equiv \operatorname{Tr}\left(e^{-\beta H+i t H}\right),
\end{gathered}
$$

where $H$ is the Hamiltonian, $\beta$ is the inverse temperature of the system, $E_{n}$ and $E_{m}$ are energy eigenvalues, and $Z(\beta)$ is the partition function of the system at $\beta . Z(\beta+i t)$ is the partition function analytically continued to the real time as $\beta \rightarrow \beta+i t$. It characterizes the discreteness of the energy spectrum. It is known that the squared quantity $\left|\frac{Z(\beta+i t)}{Z(\beta)}\right|^{2}$ is diagnostic of the pair correlation of energy eigenvalues. This squared quantity is called the spectrum form factor (see [14], for example). The characteristic behavior of the spectrum form factor in a chaotic system is that it initially drops (slope) to the minimum (dip) at the Thouless time, starts increasing (ramp) and finally approaches the constant value (plateau) after the Heisenberg time. The value of spectrum form factor after the Heisenberg time is approximated by the long timeaveraged value.

Here, we propose a subergion generalization of the spectrum form factor by using the pseudoentropy [15]. We refer the reader to [16-18] for other generalizations of the spectrum form factor. The pseudoentropy is a generalization of the entanglement entropy to a postselection process. Let $|\psi\rangle$ and $|\varphi\rangle$ be a pure state in a bipartite Hilbert space $\mathcal{H}_{A} \otimes \mathcal{H}_{\bar{A}}$. We introduce a transition matrix, 


$$
\mathcal{T}^{\psi \mid \varphi}=\frac{|\psi\rangle\langle\varphi|}{\langle\varphi \mid \psi\rangle},
$$

and a reduced transition matrix,

$$
\mathcal{T}_{A}^{\psi \mid \varphi}=\operatorname{Tr}_{\bar{A}} \mathcal{T}^{\psi \mid \varphi} .
$$

Note that (3) and (4) are two-state generalizations of the density matrix and the reduced density matrix. In particular, these transition matrices can appear naturally in the postselected process, where $|\psi\rangle$ is the initial state and $|\varphi\rangle$ is the final state. Then, we define the pseudoentropy as follows:

$$
S\left(\mathcal{T}_{A}^{\psi \mid \varphi}\right)=-\operatorname{Tr}_{A} \mathcal{T}_{A}^{\psi \mid \varphi} \log \mathcal{T}_{A}^{\psi \mid \varphi} .
$$

In the rest of this paper, we specify the initial and final states as unquenched and quenched thermofield double (TFD) states

$$
\begin{gathered}
|\psi\rangle=Z^{-\frac{1}{2}(\beta)} \sum_{n} e^{-\frac{\beta}{4}\left(H_{L}+H_{R}\right)}\left|n_{L}\right\rangle \otimes\left|n_{R}\right\rangle, \\
|\varphi\rangle=e^{-\frac{i t}{2}\left(H_{L}+H_{R}\right)}|\psi\rangle,
\end{gathered}
$$

and study the time dependence of the pseudoentropy in two-dimensional CFTs. Here the Hamiltonian $H_{L, R}$ acts on the states on $\mathcal{H}_{L, R}$, and $\left|n_{L, R}\right\rangle$ is an eigenstate of $H_{L, R}$. In Sec. II, we will clarify more explicitly the relation between the pseudoentropy for these states and the spectral form factor. The pseudoentropy is complex valued in general, as the (reduced) transition matrix $\mathcal{T}_{A}^{\psi \mid \varphi}$ is in general nonHermitian. However, the pseudoentropy can be interpreted quantum information theoretically as the number of distillable Einstein-Podolsky-Rosen pairs in the postselection process [15]. Furthermore, the pseudoentropy has a sharp gravity dual in the light of AdS/CFT [15], and can be used to detect whether two given states $|\psi\rangle$ and $|\varphi\rangle$ belong to the same quantum phase [19-21]. Throughout this paper, we normalize all dimensionful parameters by a lattice spacing.

\section{A. Summary}

We have studied the time evolution of pseudoentropy for a single interval, and our findings can be summarized as follows:

(i) The time evolution of $\operatorname{Re}\left[S_{A}\right] \equiv \operatorname{Re}\left[S\left(\mathcal{T}_{A}^{\psi \mid \varphi}\right)\right]$ is similar to that of the spectral form factor (see Fig. 2): the time evolution of $\operatorname{Re}\left[S_{A}\right]$ is characterized by two timescales, the subsystem Thouless time $t_{T}$ and Heisenberg time $t_{H}$. In particular, these are characterized by the subsystem size $\ell$ as $t_{T} \propto \sqrt{\beta \ell}$ and $t_{H} \propto \ell$ [22]. Note that the standard Thouless and Heisenberg times in the original spectral form factor are similarly characterized by the total system size $L$, instead of $\ell$. (ii) At initial time $t=0, \operatorname{Re}\left[S_{A}\right]$ is given by the entanglement entropy of the thermal state. In $0<t<t_{T}$, $\operatorname{Re}\left[S_{A}\right]$ decreases. Then, in $t_{T}<t<t_{H}, \operatorname{Re}\left[S_{A}\right]$ increases. Finally, in $t>t_{H}, \operatorname{Re}\left[S_{A}\right]$ becomes approximately constant. The constant value is approximately given by the entanglement entropy of the ground state of two-dimensional CFT Hamiltonian. In other words, the real part of the pseudoentropy exhibits a dynamical volume-area-law phase transition [23-25].

(iii) At times considerably larger than the subsystem Heisenberg time, $\operatorname{Re}\left[S_{A}\right]$ is approximately given by its long-time averaged value.

(iv) Our results reflect the expected features of entanglement spectrum in CFTs: our result in the infinite volume system shows the universal behavior for arbitrary two-dimensional CFTs. We also studied the finite size effects for the two-dimensional critical Ising model and the holographic CFTs. For the critical Ising model, we observed an erratic oscillation after the subsystem Heisenberg time $t_{H}$, while the holographic CFTs exhibited a self-averaging result.

We will also discuss how our pseudoentropy can be related to the original spectrum form factor. For these reasons, we propose that pseudoentropy can be regarded as a subregion generalization of the spectrum form factor that characterizes the entanglement spectrum.

\section{SPECTRUM FORM FACTOR FROM PSEUDOENTROPY}

In this section, we elaborate on the connection between the spectrum form factor and pseudoentropy for the TFD states in an explicit example, a two-dimensional CFT. By taking the trace of the left Hilbert space $\mathcal{H}_{L}$, we obtain

$$
\mathcal{T}_{R}^{\psi \mid \varphi}=\operatorname{Tr}_{L} \mathcal{T}^{\psi \mid \varphi}=\frac{\mathrm{e}^{-(\beta+i t) H_{R}}}{Z(\beta+i t)}
$$

The above expression (8) is already suggestive of the spectral form factor if we focus on the real part. We elaborate on this in the following. First, the pseudoentropy for $\mathcal{T}_{R}^{\psi \mid \varphi}$ is given by

$$
S\left(\mathcal{T}_{R}^{\psi \mid \varphi}\right)=(\beta+i t)\left\langle H_{R}\right\rangle_{\beta+i t}+\log Z(\beta+i t),
$$

where $\left\langle H_{R}\right\rangle_{\beta+i t}$ is the thermal expectation value of energy $H_{R}$ with complexified inverse temperature $\beta+i t$. If we consider a two-dimensional CFT, then we obtain an explicit expression for the first term as follows:

$$
\left\langle H_{R}\right\rangle_{\beta}=\frac{c \pi^{2}}{3} \frac{L_{R}}{\beta^{2}},
$$

where $L_{R}$ is the volume of the system. Considering the real part of (9), we obtain 


$$
\operatorname{Re}\left[S\left(\mathcal{T}_{R}^{\psi \mid \varphi}\right)\right]=\frac{c \pi^{2}}{3} \frac{\beta L_{R}}{\beta^{2}+t^{2}}+\frac{1}{2} \log |Z(\beta+i t)|^{2}
$$

which provides the late-time behavior of the spectrum form factor as the first term is negligible.

In the following section, we will generalize it to subsystems. Namely, we will further trace over subsystem of $R$ and call the remaining subregion as $A$. In other words, we divide the entire Hilbert space $\mathcal{H} \equiv \mathcal{H}_{L} \otimes \mathcal{H}_{R}$ into $\mathcal{H}=$ $\mathcal{H}_{A} \otimes \mathcal{H}_{\bar{A}}$ such that $A \subset R$. Our main claim is that the pseudoentropy of $\mathcal{T}_{A}^{\psi \mid \varphi}$ can be interpreted as a subregion generalization of the spectral form factor.

\section{PSEUDOENTROPY IN A TWO-DIMENSIONAL CFT}

In this section, we will present a few results regarding the pseudoentropy for the reduced transition matrix

$$
\mathcal{T}_{A}^{\psi \mid \varphi}=\operatorname{Tr}_{\bar{A}} \mathcal{T}^{\psi \mid \varphi} .
$$

Here, we divide the whole system into $A=\left[X_{1}, X_{2}\right]$ in the right system and its complement. Using the replica trick [26], the pseudoentropy can be computed as $n \rightarrow 1$ limit of the (pseudo-)Rényi entropy,

$$
S\left(\mathcal{T}_{A}^{\psi \mid \varphi}\right)=\lim _{n \rightarrow 1} S^{(n)}\left(\mathcal{T}_{A}^{\psi \mid \varphi}\right),
$$

where we introduced the (pseudo-)Rényi entropy,

$$
S^{(n)}\left(\mathcal{T}_{A}^{\psi \mid \varphi}\right)=\frac{1}{1-n} \log \operatorname{Tr}_{A}\left(\mathcal{T}_{A}^{\psi \mid \varphi}\right)^{n} .
$$

To compute the Rényi entropy, we first consider the transition matrix $\mathcal{T}^{\psi \mid \varphi}$ for the initial and the final states $|\psi\rangle,|\varphi\rangle$, prepared by the Euclidean path integral over $\mathbb{R} \times$ $\left[0, \tau_{A}\right]$ and $\mathbb{R} \times\left[0, \tau_{B}\right]$, respectively,

$$
\begin{aligned}
& |\psi\rangle=Z^{-\frac{1}{2}}\left(\tau_{A}\right) \sum_{n} \mathrm{e}^{-\tau_{A} E_{n}}\left|n_{L} n_{R}\right\rangle, \\
& |\varphi\rangle=Z^{-\frac{1}{2}}\left(\tau_{B}\right) \sum_{n} \mathrm{e}^{-\tau_{B} E_{n}}\left|n_{L} n_{R}\right\rangle .
\end{aligned}
$$

We will finally perform the Lorentzian continuation $\tau_{A}=\beta / 2, \tau_{B}=\beta / 2+i t$ after the computations to recover the original initial and the final states in real time. The reduced transition matrix $\mathcal{T}_{A}^{\psi \mid \varphi}$ for these states are given by the Euclidean path-integral on a cylinder with circumference $\tau_{A}+\tau_{B}$ with a cut on $A$ in the right system (see Fig. 1). This is explicitly given by

$$
\mathcal{T}_{A}^{\psi \mid \varphi}=\mathcal{N} \operatorname{Tr}_{\bar{A}} \sum_{n, m} e^{-\tau_{A} E_{n}}\left|n_{L} n_{R}\right\rangle\left\langle m_{L} m_{R}\right| e^{-\tau_{B} E_{m}},
$$

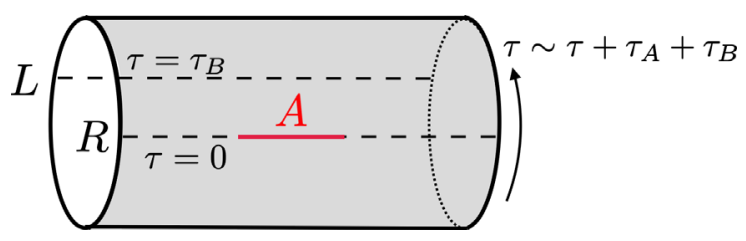

FIG. 1. The Euclidean path integral for the reduced transition matrix $\mathcal{T}_{A}^{\psi \mid \varphi}$ is performed on the Euclidean cylinder with circumference $\tau_{A}+\tau_{B}$ with a cut on $\mathrm{A}$.

where $\mathcal{N}=Z\left(\tau_{A}+\tau_{B}\right)^{-1}$. The Rényi entropy can be obtained from $\operatorname{Tr}_{A}\left(\mathcal{T}_{A}^{\psi \mid \varphi}\right)^{n}$ computed by a path integral for $n$ copies of the cylinder glued along the interval $A$. In the twist operator formalism, this amounts to computing the correlation function of twist operators with dimension $\Delta=$ $\frac{c}{12}(n-1 / n)$ on a single copy of the cylinder in an orbifold theory $\mathcal{T}^{n} / \mathbb{Z}_{n}$

$$
\begin{aligned}
S^{(n)}\left(\mathcal{T}_{A}^{\psi \mid \varphi}\right) & =\frac{1}{1-n} \log \left\langle\sigma_{n}\left(X_{1}\right) \tilde{\sigma}_{n}\left(X_{2}\right)\right\rangle, \\
& =\frac{1}{1-n} \log \left\langle\sigma_{n}\left(z_{1}, \bar{z}_{1}\right) \tilde{\sigma}_{n}\left(z_{2}, \bar{z}_{2}\right)\right\rangle .
\end{aligned}
$$

Here, we perform the exponential map $z_{i}=e^{\frac{2 \pi\left(X_{i}+i \tau\right)}{\tau_{A}+\tau_{B}}}$ from the cylinder with circumstance $\tau_{A}+\tau_{B}$ to a plane. Using the conformal symmetry, we can evaluate the two-point function of the twist operators. Finally performing the Lorentzian continuation $\tau_{A}=\beta / 2, \tau_{B}=\beta / 2+i$ to recover the original transition matrix in real time and taking the limit $n \rightarrow 1$, we obtain the following form of the pseudoentropy

$$
S\left(\mathcal{T}_{A}^{\psi \mid \varphi}\right)=\frac{c}{6} \log \frac{(\beta+i t)^{2}}{\pi^{2}} \sinh ^{2} \frac{\pi}{\beta+i t}\left(X_{1}-X_{2}\right) .
$$

Now, we consider the time dependence of the real part of the pseudoentropy (see Fig. 2). At the initial time $t=0$, the pseudoentropy is equal to the entanglement entropy for a single interval in the thermal state with inverse temperature $\beta$, as expected. Next, we focus on the late-time regime $\beta \ll t$, which has relevance to our main interest. Expanding (19) in $\beta$, we obtain

$$
S\left(\mathcal{T}_{A}^{\psi \mid \varphi}\right) \sim \frac{c}{6} \log \frac{t^{2}}{\pi^{2}} \sin ^{2} \frac{\pi}{t}\left(X_{1}-X_{2}\right)
$$

at the leading order in $\beta$. As we can approximate $\sin \frac{\pi}{t}\left(X_{1}-\right.$ $\left.X_{2}\right) \sim \frac{\pi}{t}\left(X_{1}-X_{2}\right)$ in the late-time limit $t \rightarrow \infty$, the pseudoentropy approaches the entanglement entropy for a single interval in the ground state.

$$
S\left(\mathcal{T}_{A}^{\psi \mid \varphi}\right) \rightarrow \frac{c}{6} \log \left(X_{1}-X_{2}\right)^{2} .
$$



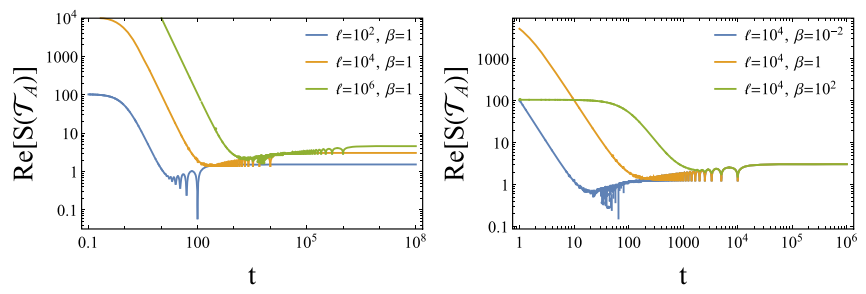

FIG. 2. Left: the time evolution of the pseudoentropy associated with two states (6) and (7) exhibits a similar behavior to the spectral form factor. Here, we consider a single interval on $R$ (let us call it $A$ ) as a subsystem. These log-log plots suggest that $t_{T} \propto \sqrt{\ell}$ and $t_{H} \propto \ell$, where $\ell \equiv\left|X_{1}-X_{2}\right|$ is the size of subsystem $A$. Right: the inverse temperature $\beta$ dependence of the pseudoentropy. These plots suggest that $t_{T} \propto \sqrt{\beta \ell}$ and $t_{H} \propto \ell$.

Therefore, while the pseudoentropy has complex values in the intermediate region, it exhibits a dynamical volumearea law phase transition [23-25]. From Fig. 2, we can summarize the time dependence of the real part of the pseudoentropy. The time evolution of the pseudoentropy starts with the entanglement entropy for the thermal states, and initially drops (slope) to a minimum (dip) at the Thouless time $t_{T} \propto \sqrt{\beta \ell}$. Note that our subsystem size $\ell$ is given by $\left|X_{1}-X_{2}\right|$. After that, it starts increasing in time (ramp) with small oscillations. Further, and at the Heisenberg time $t_{H}=\ell$, it connects to the plateau region [27]. Finally, it approaches the entanglement entropy for the ground state.

\section{TIME AVERAGE}

In this section, we discuss the late-time behaviors of the pseudoentropy and weak values, i.e., the correlation function with respect to transition matrices. In particular, we will see that they are given by the long-time-averaged values of the pseudoentropy and the weak values, and reduce to the entanglement entropy and expectation values for the vacuum state, respectively.

As a simple example, we start from a two-point function of scalar primary operators in a two-dimensional CFT,

$$
\operatorname{Tr}\left[\mathcal{T}^{\psi \mid \varphi} \mathcal{O}\left(X_{1}\right) \mathcal{O}\left(X_{2}\right)\right]=\left\langle\mathcal{O}\left(X_{1}\right) \mathcal{O}\left(X_{2}\right)\right\rangle_{\beta+i t}
$$

Here, the right-hand side is the thermal expectation value of local operators with the complexified temperature $\beta+i t$. In other words, we used the same trick as the pseudoentropy. This two point function (on a thermal cylinder) is fixed by the conformal symmetry,

$$
\left\langle\mathcal{O}\left(X_{1}\right) \mathcal{O}\left(X_{2}\right)\right\rangle_{\beta}=\left|\frac{\pi / \beta}{\sinh \left(\pi\left(X_{1}-X_{2}\right) / \beta\right)}\right|^{2 \Delta},
$$

where $\Delta$ is the scaling dimension of the scalar operator. Based on these facts, we can easily show

$$
\begin{aligned}
\lim _{T \rightarrow \infty} & \frac{1}{T} \int_{0}^{T} d t\left\langle\mathcal{O}\left(X_{1}\right) \mathcal{O}\left(X_{2}\right)\right\rangle_{\beta+i t} \\
& =\lim _{t \rightarrow \infty}\left\langle\mathcal{O}\left(X_{1}\right) \mathcal{O}\left(X_{2}\right)\right\rangle_{\beta+i t}, \\
& =\frac{1}{\left|X_{1}-X_{2}\right|^{2 \Delta}} .
\end{aligned}
$$

Here, we assumed that $X_{1}$ and $X_{2}$ are on the same time slice. The first equality (24) is obvious from the second one (25) as it converges to a time-independent value. Notice that the final expression is given by the vacuum expectation value. This example also explains the late time limit of our pseudoentropy, because it was also given by the two-point function of the twist operators, which are the (scalar) primaries on the orbifold CFT.

\section{FINITE SIZE EFFECTS}

We found that the pseudoentropy for the TFD states behaves as the subsystem generalization of the spectrum form factor. This result is universal for a two-dimensional CFT. Furthermore, the late-time behavior exactly matches its time averaging. A natural question is why we obtained such a theory-independent result. The answer is simply because we considered the entanglement spectrum associated with a single interval in an infinite volume system. In this case, the entanglement entropy provides a universal result for any twodimensional CFTs (up to the value of the central charge). In other words, the entanglement spectrum is universal [28].

In this section, we consider two finite volume systems where the entanglement spectra of our interest depend on the details of the CFT.

\section{A. Critical Ising model}

As a concrete example, we study the critical Ising model. We can numerically study the same pseudoentropy in the finite volume system. This can be implemented by the correlator method developed in $[19,20]$. We plot Figs. 3 and 4.

In Fig. 3, we observe that the oscillation becomes more suppressed as the size of total system $L$ increases. At a very late time after $t \sim L$, we have oscillations with amplitudes close to the initial value. This effect is expected to be a

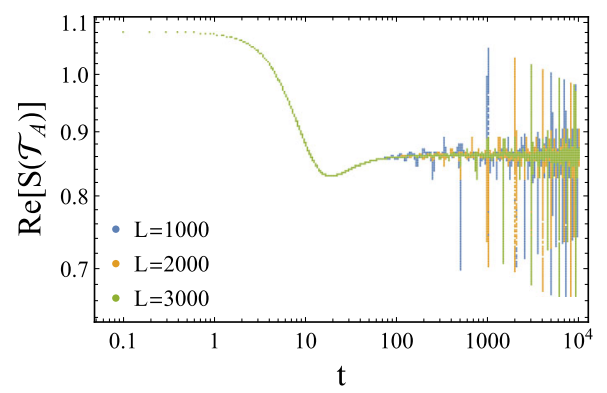

FIG. 3. Numerical results for critical Ising model with various total system sizes. Here, we set $\beta=10$ and $\ell=10$ for each plot. 


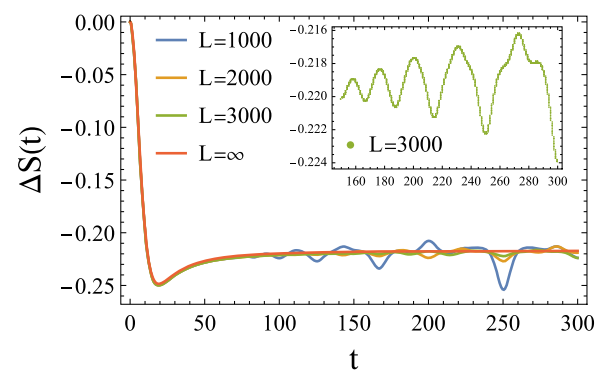

FIG. 4. Critical Ising model for a finite size system versus that for an infinite size system. Here, we plotted the subtracted pseudoentropy $\Delta S(t) \equiv \operatorname{Re}\left[S\left(\mathcal{T}_{A}^{\psi \mid \varphi}\right)(t)-S\left(\mathcal{T}_{A}^{\psi \mid \varphi}\right)(0)\right]$, for comparison. We set $\beta=10$ and $\ell=10$ for each plot. The red curve corresponds to the analytical result for an infinite-size system. Evidently, as the total system increases, the oscillation becomes smaller. The inset shows smaller erratic oscillations for $L=3000$.

consequence of the fact that the critical Ising model is integrable. We can also confirm such a large amplitude from the analytic expression of Rényi entropies [29,30]. As a consistency check, we confirmed that for larger systems, we can reproduce the results closer to analytic results in the infinite space, as shown in Fig. 4.

\section{B. Holographic CFT}

On the other hand, based on the holographic formula for pseudoentropy [15], we can apply the same analytical results as (19), when the subsystem is less than half of the total system size. This can be interpreted from the fact that the minimal surfaces do not probe the global structure when the subsystem size is not large [31,32].

In this regard, the late-time behavior of the holographic calculation is indeed self-averaging. This nature of semiclassical gravity is consistent with the original spectral form factor, which has recently attracted significant attention [12,33-35].

\section{DISCUSSION}

We proposed a generalization of the spectral form factor to subregions. Several attempts have been made in this direction previously [16-18]. In particular, the subergion Thouless and Heisenberg times defined in [16,17] depend on both the subregion and the total system size. In the future, the origin of this difference should be investigated.

Here, we only discussed the real part of the pseudoentropy, while the interpretation of the imaginary part remains unclear. An interesting future prospect would be to elucidate the role of the imaginary part.

We also discussed the holographic formula of pseudoentropy, which yields a self-averaging result, as expected from recent developments in gravity as ensemble averaging [12,33-35]. Interestingly, this behavior can be explained by using a single but complexified geometry in the bulk. We would like to stress that this behavior is also consistent with the standard CFT calculation. Notice that we needed to take into account the nonperturbative effects to probe the ramp and plateau regions in the original spectrum form factor from the holographic analysis. Perhaps such a complex geometry can play a role of an instantonlike solution, which accounts for a certain transition between different spacetime backgrounds, as in our present example. Another interesting future prospect is to investigate the role of such complexified geometries in more general contexts. In particular, it could help to generalize the standard holographic arguments to non-Hermitian systems. In this regard, it is worth pointing out that the entanglement entropy in non-Hermitian systems can also be interpreted as a version of the pseudoentropy [36]. Therefore, we expect better understanding of pseudoentropy itself would also pave the way in this direction.

\section{ACKNOWLEDGMENTS}

We thank Ali Mollabashi, Shinsei Ryu, Noburo Shiba, Kazuaki Takasan, Tadashi Takayanagi, and Zixia Wei for fruitful discussion. K. G. is supported by JSPS Grant-inAid for Early-Career Scientists 21K13930. M. N. is supported by JSPS Grant-in-Aid for Early-Career Scientists 19K14724. K. T. is supported by JSPS Grantin-Aid for Early-Career Scientists 21K13920.
[1] J. Maldacena, The large-N limit of superconformal field theories and supergravity, Int. J. Theor. Phys. 38, 1113 (1999).

[2] S. Ryu and T. Takayanagi, Holographic Derivation of Entanglement Entropy from the anti-de Sitter Space/ Conformal Field Theory Correspondence, Phys. Rev. Lett. 96, 181602 (2006).

[3] S. Ryu and T. Takayanagi, Aspects of holographic entanglement entropy, J. High Energy Phys. 08 (2006) 045.
[4] P. Calabrese and J. Cardy, Evolution of entanglement entropy in one-dimensional systems, J. Stat. Mech. (2005) P04010.

[5] P. Calabrese and J. Cardy, Entanglement and correlation functions following a local quench: A conformal field theory approach, J. Stat. Mech. (2007) P10004.

[6] P. Hosur, X.-L. Qi, D. A. Roberts, and B. Yoshida, Chaos in quantum channels, J. High Energy Phys. 02 (2016) 004. 
[7] L. Nie, M. Nozaki, S. Ryu, and M. T. Tan, Signature of quantum chaos in operator entanglement in $2 \mathrm{~d}$ CFTs, J. Stat. Mech. (2019) 093107.

[8] J. Kudler-Flam, M. Nozaki, S. Ryu, and M. T. Tan, Quantum vs classical information: Operator negativity as a probe of scrambling, J. High Energy Phys. 01 (2020) 031.

[9] H. Li and F. D. M. Haldane, Entanglement Spectrum as a Generalization of Entanglement Entropy: Identification of Topological Order in Non-Abelian Fractional Quantum Hall Effect States, Phys. Rev. Lett. 101, 010504 (2008).

[10] F. Pollmann, A. M. Turner, E. Berg, and M. Oshikawa, Entanglement spectrum of a topological phase in one dimension, Phys. Rev. B 81, 064439 (2010).

[11] H. Casini, M. Huerta, and R. C. Myers, Towards a derivation of holographic entanglement entropy, J. High Energy Phys. 05 (2011) 036.

[12] J. S. Cotler, G. Gur-Ari, M. Hanada, J. Polchinski, P. Saad, S. H. Shenker, D. Stanford, A. Streicher, and M. Tezuka, Black holes and random matrices, J. High Energy Phys. 05 (2017) 118.

[13] K. Papadodimas and S. Raju, Local Operators in the Eternal Black Hole, Phys. Rev. Lett. 115, 211601 (2015).

[14] M. L. Mehta, Random Matrices (Elsevier, New York, 2004).

[15] Y. Nakata, T. Takayanagi, Y. Taki, K. Tamaoka, and Z. Wei, New holographic generalization of entanglement entropy, Phys. Rev. D 103, 026005 (2021).

[16] X. Chen and A. W. W. Ludwig, Universal spectral correlations in the chaotic wave function, and the development of quantum chaos, Phys. Rev. B 98, 064309 (2018).

[17] X. Chen and T. Zhou, Operator scrambling and quantum chaos (2018).

[18] C.-T. Ma and C.-H. Wu, Quantum entanglement and spectral form factor (2020).

[19] A. Mollabashi, N. Shiba, T. Takayanagi, K. Tamaoka, and Z. Wei, Pseudo Entropy in Free Quantum Field Theories, Phys. Rev. Lett. 126, 081601 (2021).

[20] A. Mollabashi, N. Shiba, T. Takayanagi, K. Tamaoka, and Z. Wei, Aspects of pseudo entropy in field theories (2021).

[21] T. Nishioka, T. Takayanagi, and Y. Taki, Topological pseudo entropy (2021).

[22] We read off the scaling of the Thouless time from the numerical plots of the real part of the pseudoentropy. Small oscillations are observed in the ramp region, and the
Heisenberg time is defined as the time at which the pseudo entropy ceases to oscillate.

[23] A. Chan, R. M. Nandkishore, M. Pretko, and G. Smith, Unitary-projective entanglement dynamics, Phys. Rev. B 99, 224307 (2019).

[24] Y. Li, X. Chen, and M. P. A. Fisher, Quantum Zeno effect and the many-body entanglement transition, Phys. Rev. B 98, 205136 (2018).

[25] B. Skinner, J. Ruhman, and A. Nahum, MeasurementInduced Phase Transitions in the Dynamics of Entanglement, Phys. Rev. X 9, 031009 (2019).

[26] P. Calabrese and J. L. Cardy, Entanglement entropy and quantum field theory, J. Stat. Mech. (2004) P06002.

[27] These timescales are also clear from the equation (19) and (pseudo2): for the subsystem Thouless time, we can see the periodic oscillation becomes relevant when the real part of inside of sinh decreases to order 1 . This matches $t \sim \sqrt{\beta \ell}$. For the subsystem Heisenberg time, we can see the periodic oscillation stops when $t$ becomes order $\ell$.

[28] P. Calabrese and A. Lefevre, Entanglement spectrum in onedimensional systems, Phys. Rev. A 78, 032329 (2008).

[29] T. Azeyanagi, T. Nishioka, and T. Takayanagi, Near extremal black hole entropy as entanglement entropy via $\mathrm{AdS}_{2} / \mathrm{CFT}_{1}$, Phys. Rev. D 77, 064005 (2008).

[30] C. P. Herzog and T. Nishioka, Entanglement entropy of a massive fermion on a torus, J. High Energy Phys. 03 (2013) 077.

[31] M. Headrick and T. Takayanagi, A holographic proof of the strong subadditivity of entanglement entropy, Phys. Rev. D 76, 106013 (2007).

[32] C. T. Asplund, A. Bernamonti, F. Galli, and T. Hartman, Holographic entanglement entropy from 2d CFT: Heavy states and local quenches, J. High Energy Phys. 02 (2015) 171.

[33] P. Saad, S. H. Shenker, and D. Stanford, A semiclassical ramp in SYK and in gravity (2018).

[34] P. Saad, S. H. Shenker, and D. Stanford, JT gravity as a matrix integral (2019).

[35] G. Penington, S. H. Shenker, D. Stanford, and Z. Yang, Replica wormholes and the black hole interior (2019).

[36] P.-Y. Chang, J.-S. You, X. Wen, and S. Ryu, Entanglement spectrum and entropy in topological non-Hermitian systems and nonunitary conformal field theory, Phys. Rev. Research 2, 033069 (2020). 